\title{
Xanthogranulomatous Pancreatitis -A Rare Case Defying Clinical, Radiological and Tumor Marker Diagnostics with a Review of Literature
}

\author{
Kavita GAUR ${ }^{1}$, Shramana MANDAL ${ }^{\text {, Nidhi MAHAJAN² }}$, Sandeep SALUJA ${ }^{3}$, Satyajit GODHI ${ }^{3}$ \\ Department of Pathology, 'GB Pant Institute of Postgraduate Medical Education and Research, Jawaharlal Nehru Marg, NEW DELHI, INDIA, \\ ${ }^{2}$ Maulana Azad Medical College, Bahadurshah Zafar Marg, NEW DELHI, INDIA, and ${ }^{3}$ Department of Gastrointestinal Surgery, GB Pant Institute of Postgraduate \\ Medical Education and Research, Jawaharlal Nehru Marg, NEW DELHI, INDIA
}

\begin{abstract}
Xanthogranulomatous inflammation of the pancreas is an extremely rare entity mimicking pancreatic neoplasia. Elevation of tumor markers has seldom been reported in xanthogranulomatous inflammation of the pancreas, rendering the diagnosis difficult and highlighting the importance of a meticulous histopathological examination. A 58-year-old male presented with severe abdominal pain and anorexia for six months. A heterogeneously enhancing solid cystic mass was detected in the pancreatic head on contrast enhanced computed tomography. Endoscopic ultrasound guided aspiration yielded fluid showing elevated levels of CA19-9 (12000 IU/ml) and CEA $(221 \mathrm{ng} / \mathrm{ml})$ with a concordant increase in the serum tumour marker levels (CA 19-9:407 IU/ml; CEA: $70 \mathrm{ng} / \mathrm{ml}$ ). Clinical, radiological and biochemical evidence favouring malignancy prompted a Whipple's procedure. A thorough histopathological examination of the specimen failed to reveal malignancy. Sheets of macrophages (CD $68+$, HAM $56+$ ), chronic inflammatory cells, fibroblasts and proliferating capillaries replacing most of the pancreatic parenchyma were seen instead, nailing the diagnosis of xanthogranulomatous inflammation of the pancreas. To the best of our knowledge this is the first report of xanthogranulomatous inflammation of the pancreas without associated malignancy displaying an elevation of serum and fluid tumour markers. After seven months of follow up, the patient is asymptomatic and is doing well.
\end{abstract}

Key Words: Pancreatitis, Xanthogranulomatous inflammation, CA 19-9

\section{INTRODUCTION}

The xanthogranulomatous process characterized by the deposition of numerous foamy histiocytes heralds clinicopathological "uncertainty" (1). Patients often present with a clinical picture simulating malignancy prompting extensive surgery. Xanthogranulomatous inflammation (XGP) in the pancreas is a rarity but assumes importance as correct diagnosis often is evasive. Of the few reported cases till date (2-14), nearly all have had normal tumor marker levels. Herein, we present the first case of XGP without any associated malignancy demonstrating an elevation of both serum and fluid tumour markers. This case highlights the importance of a meticulous histopathological examination and the possible caveats of tumour marker diagnostics. Existing literature has also been reviewed to shed more light on the biological behavior of this entity.

\section{CASE REPORT}

A 58-year-old male presented with abdominal pain and weight loss for the past six months. There was no history of jaundice, gastrointestinal bleed or alcohol intake. Physical

(Turk Patoloji Derg 2019, 35:144-150)

Received : 16.08.2016 Accepted : 18.12.2016 examination was unremarkable. Laboratory investigations showed reduced values of haemoglobin $(11.3 \mathrm{~g} / \mathrm{dl})$, total protein $(4.7 \mathrm{~g} / \mathrm{dl})$ and albumin $(2.3 \mathrm{~g} / \mathrm{dl})$. A mild elevation of aspartate aminotransferase (AST) (51 IU/L) was noted. Other routine parameters including the lipid profile were within normal limits. C-reactive protein was $6.2 \mathrm{mg} / \mathrm{dl}$.

Abdominal ultrasound displayed a solid cystic lesion at the pancreatic head measuring $4.2 \times 3.9 \mathrm{cms}$. Contrast enhanced computed tomography (CECT) revealed a heterogeneously enhancing mass accompanied by dilatation of the pancreatic ducts. No calcification or stricture was noted in the main pancreatic duct (MPD). The duodenal wall surrounding the pancreas was thickened (Figure 1A,B). A diagnosis of malignancy at the head of the pancreas with focal chronic pancreatitis was suggested.

Endoscopic ultrasound (EUS) guided aspiration guided aspiration yielded fluid displaying a supranormal elevation of CA 19-9(12000 IU/ml). Carcinoembryonic antigen $(\mathrm{CEA})(221 \mathrm{ng} / \mathrm{ml})$ and fluid amylase $(5419 \mathrm{IU} / \mathrm{ml})$ were also raised. The serum levels of CA 19-9 and CEA were also elevated (407.36 IU/ml and $70 \mathrm{ng} / \mathrm{ml}$, respectively). Serum

Correspondence: Shramana MANDAL

GB Pant Institute of Postgraduate Medical Education and Research and Associated Maulana Azad Medical College, Department of Pathology, NEW DELHI, INDIA

E-mail: shra_mana@hotmail.com Phone: +91 119968604254 
lipase and amylase, were within normal limits. A strong clinical suspicion of malignancy supported by imaging and biochemical findings culminated in a Whipple's pancreaticoduodenectomy. Per-operative findings showed a bulky pancreatic head with a palpable cystic lesion. The rest of the pancreas appeared firm and the MPD was dilated.

Gross examination of the specimen revealed a solid and cystic lesion in the head of the pancreas measuring $6.1 \mathrm{x}$ $5.2 \times 4.0 \mathrm{~cm}$. The solid area was firm and yellow in colour (Figure 2A). Clear, non-viscous yellow fluid was noted in the cystic area. Microscopic examination showed dense inflammatory infiltrate composed of foamy histiocytes, hemosiderin laden macrophages and bile pigment largely replacing the normal pancreatic parenchyma and extending upto the duodenal mucosa with splaying of the muscularis mucosa (Figure 2B-D). Also seen were dilated pancreatic ducts with inspissated secretions (Figure 3A). Cholesterol clefts and foreign body giant cells were interspersed (Figure 3B,C). Extensive sectioning and complete exhaustion of the tissue showed no focus of malignancy. Pan-cytokeratin (CK) and epithelial membranous antigen (EMA) were employed in the sections to rule out any neoplastic epithelial cells. Both were negative. The sheets of macrophages were diffusely positive for anti macrophage markers CD 68 and HAM 56 (Figure 3D). Thus a final diagnosis of XGP was made. The patient is under follow up and shows no features of recurrence after seven months. C reactive protein is near normal $(3.1 \mathrm{mg} / \mathrm{dl})$. Follow up serum CA 19-9 \& CEA have shown a reduction, though both have not touched the baseline reference values (CA 19-9:71.16, CEA: $9.86 \mathrm{ng} /$ $\mathrm{ml})$.

\section{DISCUSSION}

Xanthogranulomatous inflammation histologically encompasses an accumulation of lipid rich histiocytes, multinucleated giant cells, plasma cells, cholesterol clefts and fibrous tissue (1). XGP is a rare entity with only a handful of reports in medical literature (2-14). Out of a total of 4500 cases of chronic cholecystitis diagnosed since 2007 at our institute xanthogranulomatous cholecystitis (XGC) comprised 353 (7.84\%). In contrast, XGP was seen in $0.74 \%$ cases $(1 / 136)$ of chronic pancreatitis in the same time frame. XGP as a distinct entity is believed to occur due to intracystic hemorrhage (2), stone formation (3), mucin leakage due to an associated tumour $(4,9)$ or local duct obstruction in the absence of precipitating factors (12). In the present case, XGP possibly occurred due to local duct damage leading to bile extravasation and subsequent xanthogranulomatous response.

To the best of our knowledge this is the only case with tumour marker elevation in the absence of any associated neoplastic process. One prior case with elevated tumour markers had an associated mucinous cystic neoplasm (13). A review of the existing literature (Table I) revealed mean age at presentation of 59.4 years with a definite male predilection (76.4\%). A distinct geographic predilection was noted with 13/14 (92.8\%) reports published from the Asian -Eurasian belt including Turkey. The most common presenting symptom of such patients is abdominal pain (66.6\%). On imaging, most cases presented as solid or cystic masses suggesting a pancreatic neoplasm. All patients underwent major surgical intervention highlighting the lacuna in picking up this form of pancreatitis preoperatively and hence the need to analyze this entity better.

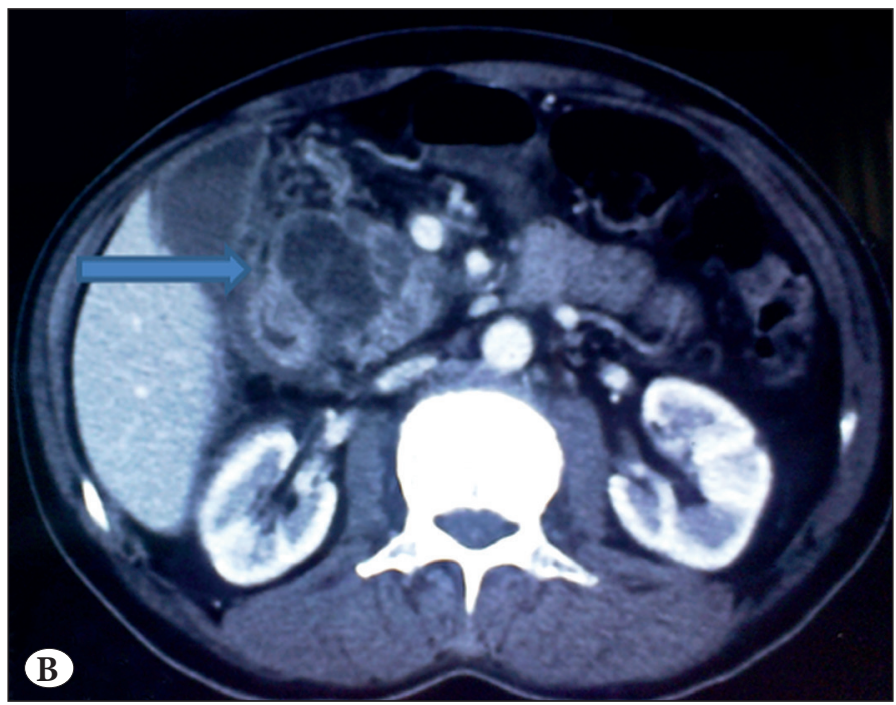

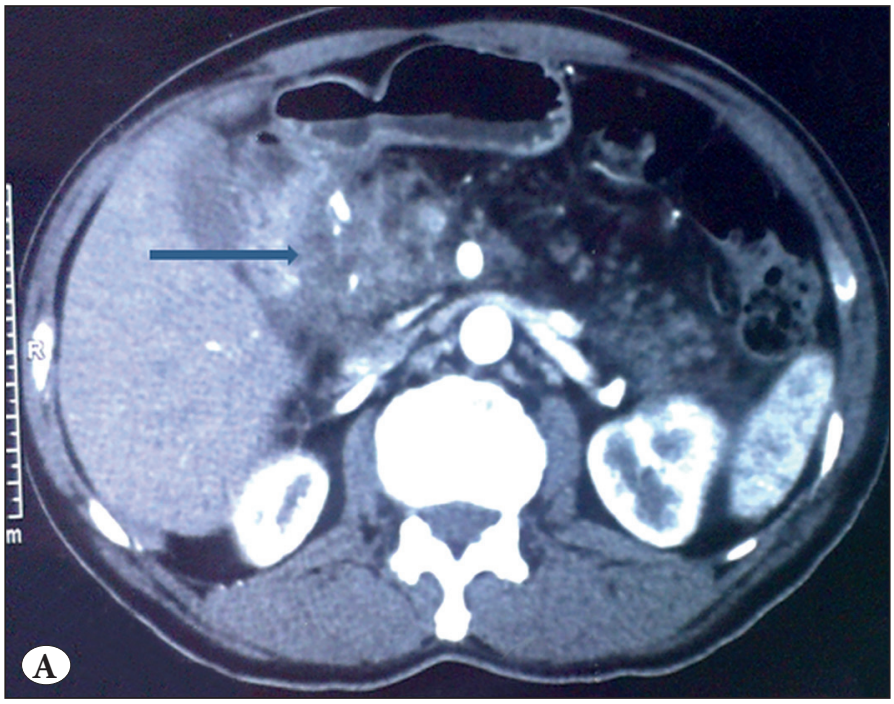

Figure 1: A,B) Axial section of shows a heterogeneous mass in head of pancreas (arrow) with a cystic component. 
Histopathological study of resected specimens remains the gold standard of diagnosis with dense sheets of foamy macrophages and giant cells in the absence of neoplastic cells providing clues to nailing this lesion. In our case extensive sectioning at multiple levels was performed to exclude a neoplastic etiology. Florid xanthogranulomatous inflammation may mask rare malignant cells entrapped in the milieu. Hence, a meticulous approach must be employed by the pathologist. An immunostain panel employing cytokeratins, EMA and macrophage markers (CD 68, HAM 56) can prevent potential error in diagnosis. Well directed peroperative frozen sectioning may also help in giving the clinicians a timely diagnosis.

This case was unique as it was accompanied by tumour marker elevation in both serum and cyst fluid. Tumour marker analysis in pancreatic malignancy largely revolves around serum measurements of the widely available CA 19-9. Unfortunately, definite pitfalls exist in its diagnostic capabilities with elevated levels of CA 19-9 also being reported in benign conditions associated including pancreatitis (15). The present case validates the American Society of Clinical Oncology (ASCO) guidelines which rule out the use of CA 19-9 for screening or diagnostic purpose (16). Lewandrowski et al. (15) in a detailed review pointed out that tumour marker elevation in cyst fluid is not pathognomic of malignancy. In fact, serous neoplasms may have perfectly normal values of tumour markers, similar to benign pancreatic pseudocysts. Imaging modalities like computerized tomography (CT), Magnetic resonance cholangiopancreaticography (MRCP) have also been unsatisfactory in picking up the xanthogranulomatous process rendering the diagnosis difficult at times (10).
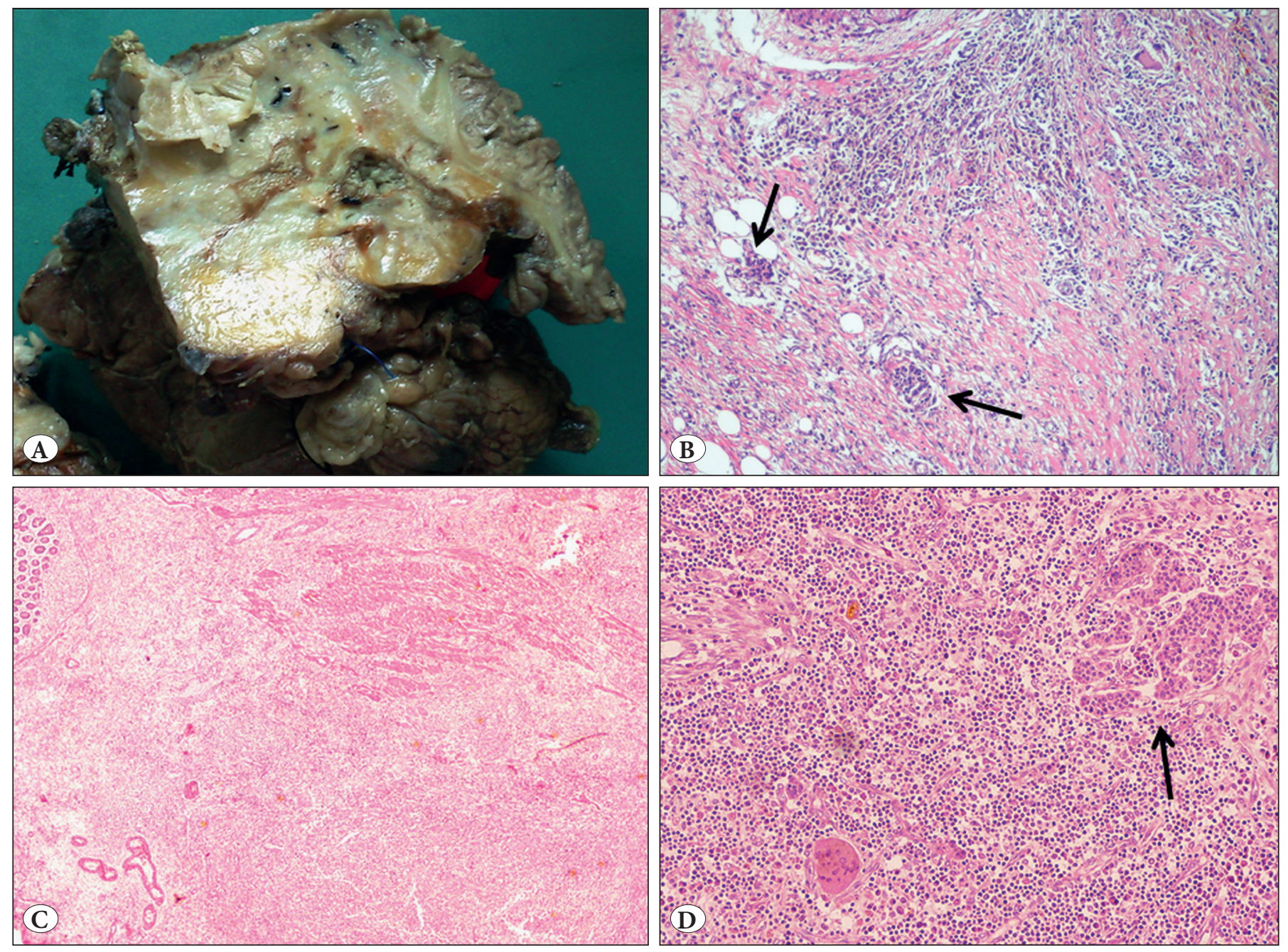

Figure 2: A) Gross photograph of the pancreas showing a solid area firm and yellow in color (arrow), red circle depicting preliminary sectioned pancreaticoduodenal groove. B) Dense inflammation replacing the normal pancreatic parenchyma. C) Inflammation extending upto the duodenal mucosa with splaying of the muscularis (H\&E; x100). D) Higher magnification shows dense inflammatory infiltrate composed of foamy histiocytes, hemosiderin laden macrophages and bile pigment (H\&E; x200). Arrows highlight residual pancreatic parenchyma in $\mathrm{B}, \mathrm{D}$. 


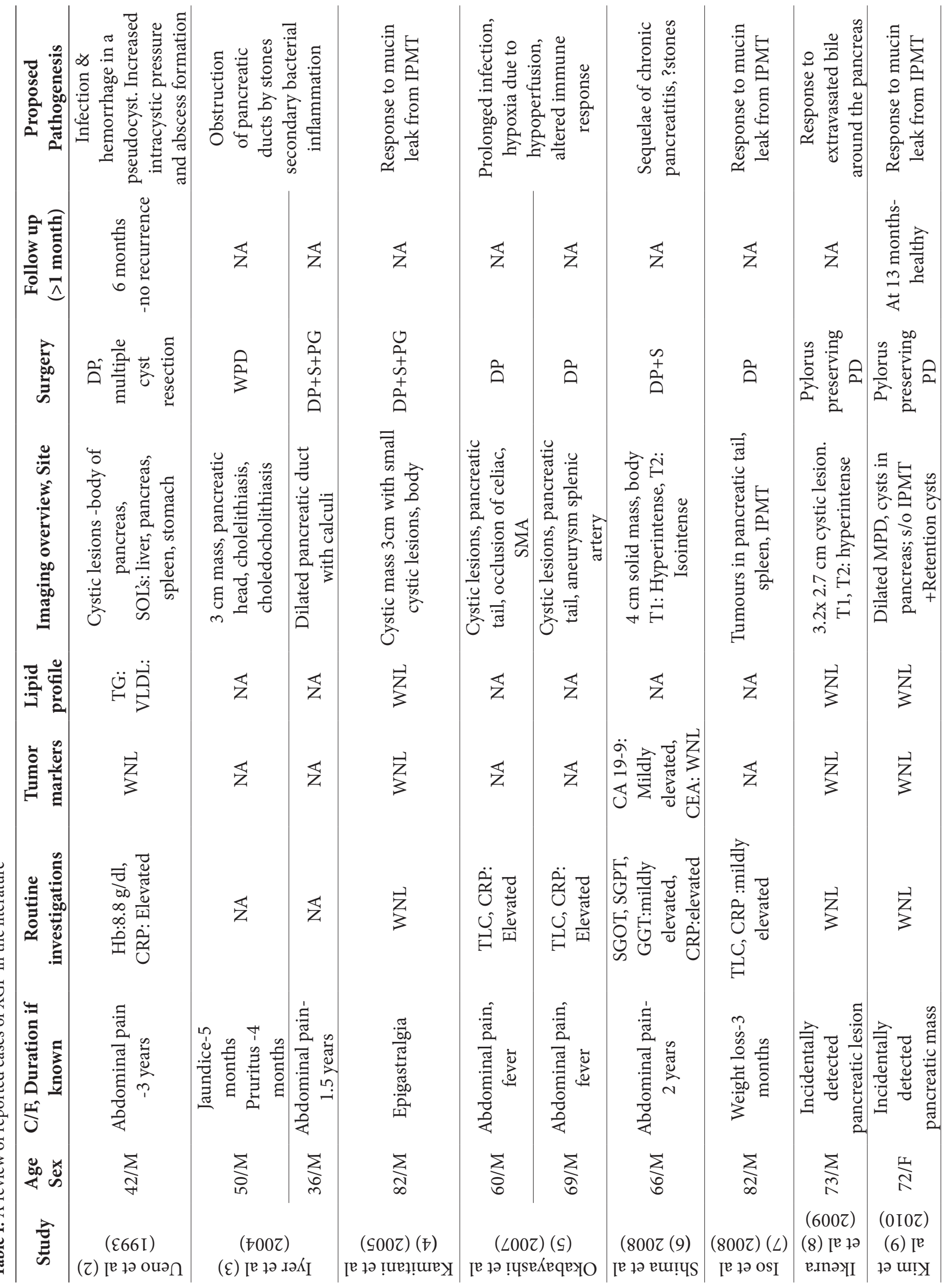




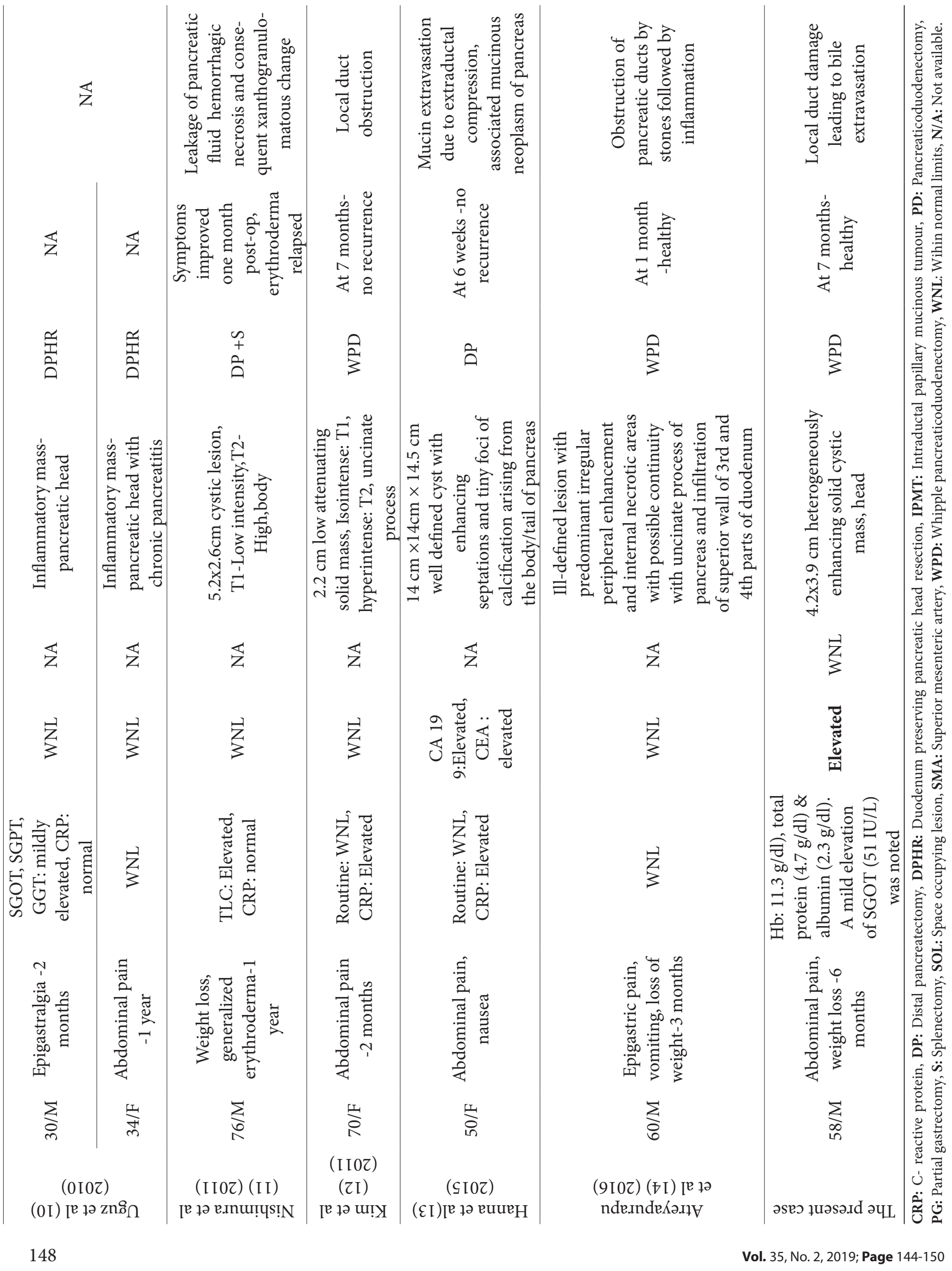


A close differential diagnosis to be considered in the present case is paraduodenal pancreatitis (PDP). Both PDP and XGP can mimic malignancy clinically and radiologically as seen in this case. Our patient did not have a history of chronic alcohol intake, a common predisposing factor for PDP. Gross examination is important to determine the site of pathology which in PDP centres at the pancreaticoduodenal groove and duodenal wall. Here, the pancreas was grossly the area of affliction and the duodenal mucosa appeared unremarkable. Histological features described in PDP (17) such as Brunner gland hyperplasia, duodenal submucosal fibrosis, cysts in the duodenal wall, heterotopic pancreatic tissue and proliferation of myoid cells were all absent in this case. Interestingly prominent chronic inflammation as seen in this case is not a feature of PDP (17). The singularly extensive presence of foamy histiocytes in areas grossly corresponding to the pancreas on the other hand rendered the diagnosis of XGP.

This case highlights that if unfavourable clinical and biochemical findings are juxtaposed, arriving at the correct diagnosis may be next to impossible before surgical resection. Frozen sections from the resected lesion used in tandem with rapid immunohistochemistry may be a useful modality in determining the extent of resection as has been suggested for the more frequently encountered XGC (18).

In conclusion, we suggest that for solid cystic/cystic lesions of the pancreas-XGP, though rare must be kept as a possible differential diagnosis. Further studies would be needed to explore a valid biomarker for xanthogranulomatous
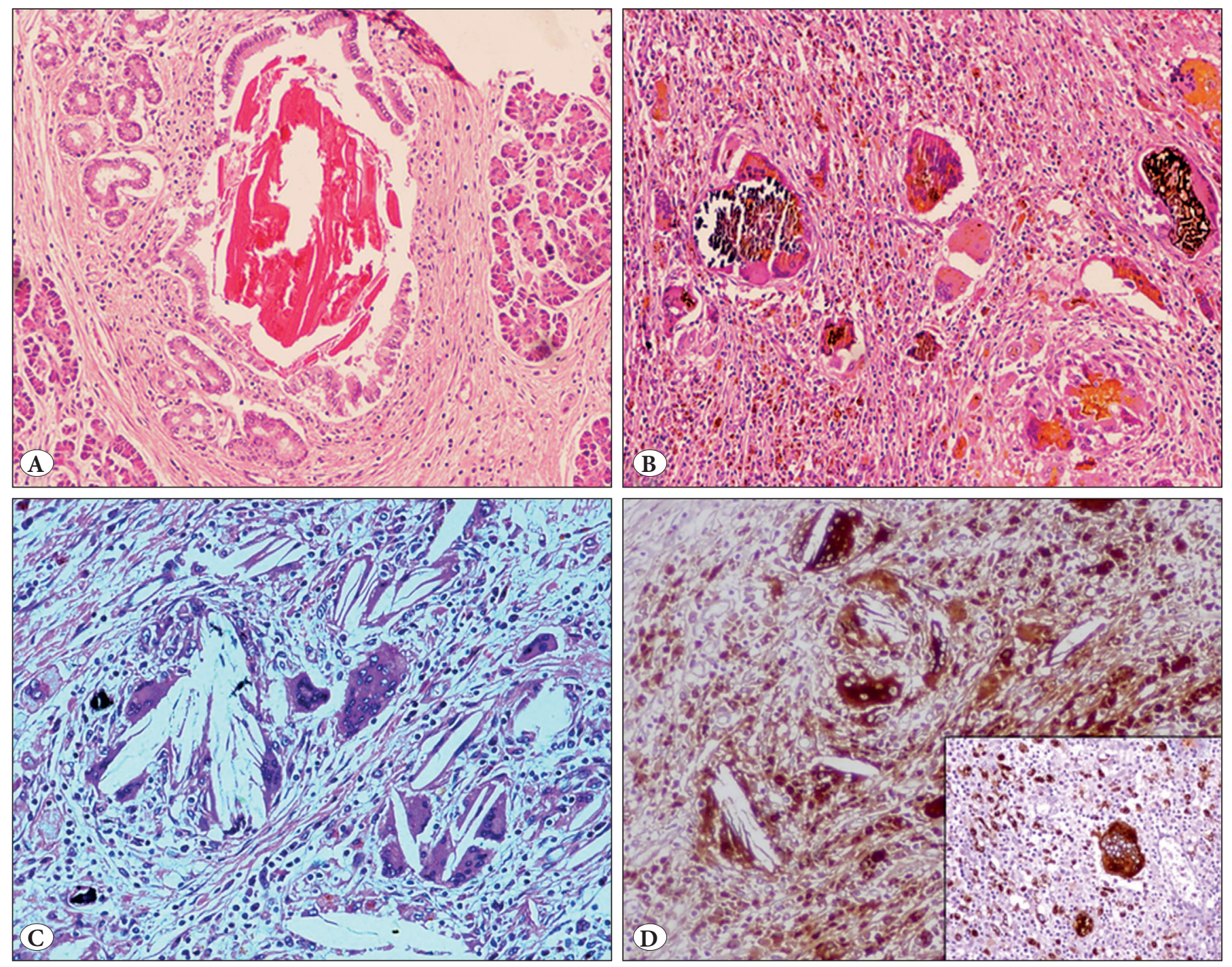

Figure 3: A) Dilated pancreatic ducts with inspissated secretions (H\&E; x200). B,C) Cholesterol clefts with foreign body giant cells (H\&E; $\mathrm{x} 200$ ). D) Immunostaining of HAM56 highlights the predominance of macrophages in the inflammatory infiltrate (HAM56; 200 ). Inset: Giant cells highlighted by HAM56 staining (x100). 
inflammation and secondly to validate the efficacy of a frozen based approach in curtailing patient morbidity thus saving patients from unnecessary extensive surgical procedures.

Ethical Approval: Informed consent has been obtained from the patient for this report and due efforts have been made to uphold patient confidentiality in the writing of the manuscript.

\section{Conflict of Interest: None}

\section{Funding/Grants/Sponsorship: None obtained}

\section{REFERENCES}

1. Cozzutto C, Carbone A. The xanthogranulomatous process. Xanthogranulomatous inflammation. Pathol Res Pract. 1988;183:395-402.

2. Ueno T, Hamanaka Y, Nishihara K, Nishida M, Nishikawa M, Kawabata A, Yamamoto S, Tsurumi M, Suzuki T. Xanthogranulomatous change appearing in the pancreas cyst wall. Pancreas. 1993;8:649-51.

3. Iyer VK, Aggarwal S, Mathur M. Xanthogranulomatous pancreatitis: Mass lesion of the pancreas simulating pancreatic carcinoma - a report of two cases. Indian J Pathol Microbiol. 2004;47:36-8.

4. Kamitani T, Nishimiya M, Takahashi N, Shida Y, Hasuo K, Koizuka $\mathrm{H}$. Xanthogranulomatous pancreatitis associated with intraductal papillarymucinous tumor. AJR Am J Roentgenol. 2005,185:704-7.

5. Okabayashi T, Nishimori I, Kobayashi M, Sugimoto T, Kohsaki T, Okamoto K, Ito S, Moriki T, Araki K, Onishi S. Xanthogranulomatous pancreatic abscess secondary to acute pancreatitis: Two case reports. Hepatogastroenterology. 2007;54:1648-51.

6. Shima Y, Saisaka Y, Furukita Y, Nishimura T, Horimi T, Nakamura T, Tanaka K, Shibuya Y, Ozaki K, Fukui Y, Hamada M, Nishioka Y, Okabayashi T, Taniki T, Morita S, Iwata J. Resected xanthogranulomatous pancreatitis. J Hepatobiliary Pancreat Surg.2008;15: 240-2.

7. Iso Y, Tagaya N, Kita J, Sawada T, Kubota K. Xanthogranulomatous lesion of the pancreas mimicking pancreatic cancer. Med Sci Monit. 2008;14: CS130-3.
8. Ikeura T, Takaoka M, Shimatani M, Koyabu M, Kusuda T, Suzuki R Sumimoto K, Okazaki K. Xanthogranulomatous inflammation of the peripancreatic region mimicking pancreatic cystic neoplasm. Intern Med. 2009;48:1881-4.

9. Kim YN, Park SY, Kim YK, Moon WS. Xanthogranulomatous pancreatitis combined with intraductal papillary mucinous carcinoma in situ. J Korean Med Sci. 2010;25:1814-7.

10. Uguz A, Yakan S, Gurcu B, Yilmaz F, Ilter T, Coker A. Xanthogranulomatous pancreatitis treated by duodenumpreserving pancreatic head resection. Hepatobiliary Pancreat Dis Int. 2010;9:216-8.

11. Nishimura M, Nishihira T, Hirose T, Ishikawa Y, Yamaoka $\mathrm{R}$, Inoue $\mathrm{H}$,Tatsuta $\mathrm{M}$. Xanthogranulomatous pancreatitis mimicking a malignant cystic tumour of the pancreas: Report of a case. Surg Today.2011;41:1310-3.

12. Kim HS, Joo M, Chang SH, Song HY, Song TJ, Seo JW, Kim CN. Xanthogranulomatous pancreatitis presents as a solid tumour mass: A case report. J Korean Med Sci. 2011;2:583-6.

13. Hanna T, Abdul-Rahman Z, Greenhalf W, Farooq A, Neoptolemos JP. Xanthogranulomatous pancreatitis associated with a mucinous cystic neoplasm. Pathol Int. 2015 Nov 12.

14. Atreyapurapu V, Keshwani A, Lingadakai R, Pai K. Xanthogranulomatous pancreatitis mimicking a malignant solid tumour. BMJ Case Rep 2016;2016.

15. Lewandrowski KB, Southern JF, Pins MR, Compton CC, Warshaw AL. Cyst fluid analysis in the differential diagnosis of pancreatic cysts. A comparison of pseudocysts, serous cystadenomas, mucinous cystic neoplasms, and mucinous cystadenocarcinoma. Ann Surg. 1993;217:41-7.

16. Locker GY, Hamilton S, Harris J, Jessup JM, Kemeny N, Macdonald JS, Somerfield MR, Hayes DF, Bast RC Jr; ASCO. ASCO 2006 update of recommendations for the use of tumor markers in gastrointestinal cancer. J Clin Oncol. 2006;24:531327.

17. DeSouza K, Nodit L. Groove pancreatitis: A brief review of a diagnostic challenge. Arch Pathol Lab Med. 2015;139:417-21.

18. Rastogi A, Singh DK, Sakhuja P, Gondal R. Florid xanthogranulomatous cholecystitis masquerading as invasive gall bladder cancer leading to extensive surgical resection. Indian J Pathol Microbiol. 2010;53:144-7. 